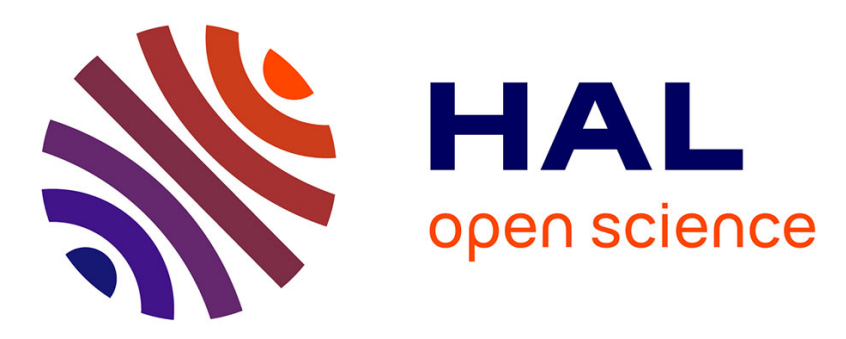

\title{
Dépendance, soins de longue durée et politiques publiques en Europe
}

Claude Martin, Blanche Le Bihan

\section{To cite this version:}

Claude Martin, Blanche Le Bihan. Dépendance, soins de longue durée et politiques publiques en Europe. PUR. Vieillesses et vieillissements. Regards sociologiques, Presses universitaires de rennes, pp.127-140, 2014, 978275352946 5. hal-01166698

\section{HAL Id: hal-01166698 \\ https://hal.science/hal-01166698}

Submitted on 23 Jun 2015

HAL is a multi-disciplinary open access archive for the deposit and dissemination of scientific research documents, whether they are published or not. The documents may come from teaching and research institutions in France or abroad, or from public or private research centers.
L'archive ouverte pluridisciplinaire HAL, est destinée au dépôt et à la diffusion de documents scientifiques de niveau recherche, publiés ou non, émanant des établissements d'enseignement et de recherche français ou étrangers, des laboratoires publics ou privés. 


\section{Dépendance, soins de longue durée et Politiques publiques en Europe}

La dépendance des personnes âgées ou les soins de longue durée représentent un nouveau défi pour les systèmes de protection sociale européens. Ce défi est à la mesure du vieillissement de la population européenne (section 1). Enjeu majeur de politiques publiques (section 2), la dépendance des personnes âgées fait aujourd'hui l'objet de mesures mixtes, combinant soutien public et contribution des familles. Dans un souci de réduction des coûts, la convergence des politiques menées dans les différents pays européens vers des dispositifs de "cash for care " (prestations monétaires) et l'introduction de congés permettant aux aidants familiaux de s'occuper de leurs parents âgés met au cœur des débats les questions de la flexibilité des arrangements de care et de l'articulation entre intervention publique et intervention familiale (section 3 ).

\section{Le vieillissement de l'Europe et ses conséquences}

Le vieillissement d'une population correspond à l'accumulation de celle-ci aux âges élevés (plus de 60 ou plus de 65 ans, selon les cas). II peut être diversement évalué. La mesure de l'âge moyen d'une population est un premier outil, encore très approximatif. Plus généralement, on apprécie le vieillissement d'une population en termes de proportion des personnes de plus de 60 ou de 65 ans dans la population générale ou bien encore, on rapporte cette population " âgée " à la population des " jeunes" (moins de 15 ou moins de 20 ans, selon les cas). Lorsqu'il est question de retraite et d'équilibre des comptes par répartition, on peut aussi rapporter les plus de 60 ou 65 ans à la population des adultes en âge de travailler et donc de cotiser (20-59 ou 20-65 ans). Deux facteurs contribuent principalement à ce vieillissement global : le niveau de fécondité et l'espérance de vie, sachant que Sauvy insistait sur ce premier facteur pour expliquer l'importance du phénomène.

On sait aujourd'hui que le vieillissement de la population européenne est principalement dû à de formidables gains d'espérance de vie. Au début du $21^{\text {ème }}$ siècle, près des deux tiers des 27 Etats membres ont une espérance de vie à la naissance supérieure à 80 ans pour les femmes et à 75 ans pour les hommes (voir tableau 1), et cette espérance de vie continue de progresser

1 . Blanche Le Bihan, politiste, enseignant-chercheur à l'EHESP, chercheur au CRAPE UMR 6051 Université de Rennes 1 ; Claude Martin, sociologue, directeur de recherche au CNRS, directeur du CRAPE UMR 6051, Université de Rennes 1-CNRS-Science Po Rennes, Chaire 'Social Care - Lien social et santé' à l'EHESP. Cet article reprend plusieurs articles déjà publiés par les auteurs (Martin, 2006 ; Le Bihan et Martin, 2010a et b). 
(approximativement de deux à trois mois par an dans la vieille Europe) $)^{2}$. Certes, les pays européens " vieillissent " à des rythmes différents : I'Allemagne, l'Italie et la Grèce "vieilliraient» ainsi plus rapidement que la France ou l'Irlande. Mais, quoiqu'il en soit, le nombre des personnes âgées de plus de 65 ans en Europe en 2010 est le double de ce qu'il était en 1960, passant de 34 à 69 millions d'individus.

Tableau 1 : Indicateur de fécondité et d'espérance de vie dans l'Union européenne (2007)

\begin{tabular}{|c|c|c|c|}
\hline & \multirow[t]{2}{*}{ Indic. conj. Fécondité (2007) } & \multicolumn{2}{|c|}{$\begin{array}{l}\text { Espérance de vie à la naissance, en } \\
\text { année (2007) }\end{array}$} \\
\hline Pays UE27 & & Hommes & Femmes \\
\hline Belgique & $1,72(2005)$ & 77,1 & 82,6 \\
\hline Danemark & 1,84 & 76,2 & 80,6 \\
\hline Allemagne & 1,37 & 77,4 & 82,7 \\
\hline Grèce & 1,41 & 77,1 & 81,8 \\
\hline Espagne & 1,40 & 77,8 & 84,3 \\
\hline France & 1,98 & 77,6 & 84,8 \\
\hline Irlande & 2,01 & 77,4 & 82,2 \\
\hline Italie & 1,37 & $78(2006)$ & $83,6(2006)$ \\
\hline Luxembourg & 1,61 & 76,7 & 82,2 \\
\hline Pays-Bas & 1,72 & 78,1 & 82,5 \\
\hline Autriche & 1,38 & 77,5 & 83,1 \\
\hline Portugal & 1,33 & 75,9 & 82,2 \\
\hline Finland & 1,83 & 76 & 83,1 \\
\hline Suède & 1,88 & 79 & 83,1 \\
\hline $\mathrm{R}-\mathrm{U}$ & 1,90 & 77,6 & 81,8 \\
\hline Chypre & 1,39 & 77,8 & 82,2 \\
\hline Rep. Tch & 1,44 & 73,8 & 80,2 \\
\hline Estonie & 1,63 & 67,2 & 78,8 \\
\hline Hongrie & 1,32 & 69,4 & 77,8 \\
\hline Lettonie & 1,41 & 65,8 & 76,5 \\
\hline Lituanie & 1,35 & 64,9 & 77,2 \\
\hline Malte & 1,37 & 77,5 & 82,2 \\
\hline Polog & 1,31 & 71 & 79,8 \\
\hline Slovaquie & 1,25 & 70,6 & 78,4 \\
\hline Slovénie & 1,38 & 74,7 & 82 \\
\hline Bulgarie & 1,42 & 69,5 & 76,7 \\
\hline
\end{tabular}

2 Rappelons qu'elle était en France de 45 ans en moyenne pour les deux sexes en 1900 et de 70 ans en 1958 et que durant la première moitié du $20^{\text {ème }}$ siècle l'essentiel des gains d'espérance de vie à la naissance était dû à la baisse de la mortalité infantile. A partir des années 1970, c'est surtout l'espérance de vie à 65 ans qui a augmenté régulièrement. 


\begin{tabular}{|c|c|c|c|}
\hline Roumanie & 1,30 & 69,7 & 76,9 \\
\hline
\end{tabular}

Source: Europe in Figures, Eurostat year book, 2010.

Il faut néanmoins se défier d'une vision simpliste de la notion d'âge. Comme l'a bien montré Patrice Bourdelais (1993), le plus important ne serait-il pas plutôt d'évaluer le temps qui nous sépare de la fin de notre existence et l'état de santé dans lequel nous approchons de cette fin, d'où la notion d'espérance de vie en bonne santé. Se pose alors le problème des inégalités sociales, de genre et enfin de territoire (Grand et al., 2000). Autant de variables qui agissent directement sur cette espérance de vie en bonne santé.

Les réactions à cette conquête de longévité sont ambivalentes. S'il s'agit incontestablement d'une conquête, la longévité suscite également des inquiétudes. D'abord en termes symboliques, comme lorsque l'on annonce que désormais, dans plusieurs pays européens, le nombre de "vieux " (plus de 60 ans) est supérieur à celui des enfants (moins de 15 ans). Le vieillissement de la population est aussi souvent considéré comme une source d'accroissement des dépenses de santé, dans la mesure où l'on observe que la demande de soins et la consommation médicale augmentent avec l'âge. L'ensemble des postes de dépenses des systèmes de santé (dépenses hospitalières, de médecine ambulatoire, de médicaments) pourraient être affectés par ce phénomène de vieillissement. D'après les estimations de la commission européenne - en pourcentage du produit intérieur brut l'accroissement des dépenses publiques de santé dû au vieillissement entre 2004 et 2050 varie entre 1 et $2 \%$ du PIB de chaque pays. Et c'est en France que, d'après ces estimations, le niveau de dépenses pourrait se révéler le plus élevé en 2050, pour atteindre près de 9,5\% du PIB (Source European Communities, 2007).

Les projections futures soulèvent un certain nombre d'interrogations et suscitent des controverses. Les plus alarmistes prévoient une augmentation de ces coûts pour les systèmes de santé liés à l'arrivée d'ici 2020 des générations du baby-boom aux âges élevés (plus de 75 ans). Mais le problème n'est pas seulement celui de l'importance numérique de ces cohortes de personnes âgées et très âgées à venir, mais aussi leurs pratiques de consommation de biens et de services. Dans la même logique, un certain nombre d'auteurs estiment que la question est plutôt celle de l'allongement de vie en bonne santé, lié au meilleur état de santé global de ces nouvelles générations de vieillards (Robine et Cheung, 2008).

\section{La dépendance comme enjeu de politique publique}

L'allongement de l'existence humaine modifie aussi la conception que l'on se faisait du cycle de vie. Si après la seconde guerre mondiale, on avait coutume de distinguer trois étapes ou trois âges de la vie (l'enfance : âge de la socialisation et de la scolarisation; l'âge adulte comme âge du travail et le troisième âge comme âge d'un repos chèrement acquis par une vie de labeur), il semble que l'on y ajoute de nouveaux âges dont doivent tenir compte les pouvoirs publics : la jeunesse, comme âge de transition prolongée vers l'âge adulte et le quatrième âge, conçu comme la période où s'accroît le risque d'être confronté aux maladies chroniques, aux incapacités et à la dépendance.

La classe d'âge qui croît le plus rapidement est précisément la plus âgée (older old) : les 80 ans et plus. Eurostat estime qu'en 2050, cette classe d'âge représentera $11,4 \%$ de l'ensemble de la population européenne (à comparer à $4 \%$ en 2005). Cet accroissement de la population des personnes très âgées pose le problème de la demande de soins et d'aide quotidienne, du fait des 
risques d'incapacités et de handicaps qui augmentent avec l'âge. Cette demande de soins est perçue comme un enjeu majeur d'action publique, et ce pour deux raisons. Non seulement le besoin de soutien et d'aide quotidienne des personnes âgées pourrait augmenter de façon considérable dans les décennies à venir, mais de plus, l'offre de soins, d'aide et de soutien fournie par les proches risque de diminuer, ce que certains experts qualifient de care deficit. En effet, dans la société de croissance de l'Après-Guerre, la quasi-totalité de l'aide aux personnes âgées souffrant d'incapacités ou de handicaps était assurée par des membres de la famille : le plus souvent des femmes, épouses, filles et brus. La participation accrue des femmes au marché du travail, d'une part, et l'instabilité des ménages qui complexifient et démultiplient potentiellement le nombre des personnes âgées apparentées, d'autre part, rendraient les femmes moins disponibles pour ce travail de soins non rémunéré. Malgré cette convergence des défis, s'opposent deux manières de le qualifier : certains pays comme la France identifient un public-cible spécifique: les personnes âgées dépendantes, quand d'autres préfèrent ne pas ériger de barrière d'âge et évoquent les politiques de " soins de longue durée » (Long-term Care).

Si l'on se réfère aux typologies des régimes d'Etat-providence (Esping-Andersen, 1990), on peut déduire quatre principaux types de réponse publique aux besoins croissants des personnes âgées dépendantes, correspondant aux quatre principaux régimes de protection sociale de la vieille Europe (voir tableau 2) (auquel il faudrait ajouter les systèmes des pays de l'Est de l'Europe des 27) :

Tableau 2 : Les politiques de Long Term Care selon les régimes d'Etat-providence

\begin{tabular}{|c|c|c|c|c|}
\hline $\begin{array}{l}\text { Les politiques de } \\
\text { "Long term } \\
\text { care »selon les } \\
\text { régimes d'Etat- } \\
\text { providence }\end{array}$ & Libéral & $\begin{array}{l}\text { Familialiste et } \\
\text { clientéliste }\end{array}$ & Corporatiste & Socio-démocrate \\
\hline $\begin{array}{l}\text { Principes dominant } \\
\text { de solidarité }\end{array}$ & Le marché & La famille & $\begin{array}{l}\text { La corporation } \\
\text { professionnelle }\end{array}$ & La citoyenneté \\
\hline Finalité du système & $\begin{array}{l}\text { Garantir la } \\
\text { couverture des } \\
\text { besoins par le } \\
\text { marché et la } \\
\text { solidarité familiale }\end{array}$ & $\begin{array}{l}\text { Définir localement } \\
\text { des services } \\
\text { complémentaires à la } \\
\text { famille }\end{array}$ & $\begin{array}{l}\text { Garantir au } \\
\text { travailleur et à sa } \\
\text { famille un niveau } \\
\text { de vie sur la base } \\
\text { de ses ressources } \\
\text { antérieures }\end{array}$ & $\begin{array}{l}\text { Promotion de la } \\
\text { citoyenneté sociale en } \\
\text { définissant des droits } \\
\text { sociaux universels }\end{array}$ \\
\hline $\begin{array}{l}\text { Principes de } \\
\text { fonctionnement }\end{array}$ & $\begin{array}{l}\text { Responsabilité } \\
\text { individuelle }\end{array}$ & \begin{tabular}{|l} 
Obligation \\
alimentaire, solidarité \\
familiale
\end{tabular} & $\begin{array}{l}\text { Contributivité des } \\
\text { travailleurs et } \\
\text { assistance pour les } \\
\text { non travailleurs }\end{array}$ & $\begin{array}{l}\text { Egalité d'accès à des } \\
\text { services collectifs } \\
\text { décentralisés }\end{array}$ \\
\hline Mode de financement & $\begin{array}{l}\text { Ressources de la } \\
\text { personne âgée } \\
\text { (désépargne) et de } \\
\text { sa famille et } \\
\text { assurance privée }\end{array}$ & \begin{tabular}{|l} 
Ressource de la \\
personne âgée plus \\
obligation \\
alimentaire élargie et \\
assistance
\end{tabular} & $\begin{array}{l}\text { Cotisation } \\
\text { obligatoire }\end{array}$ & $\begin{array}{l}\text { Impôts nationaux et } \\
\text { locaux }\end{array}$ \\
\hline
\end{tabular}




\begin{tabular}{|l|l|l|l|l|}
\hline Offre de services & $\begin{array}{l}\text { Dominée par } \\
\text { industrie privée } \\
\text { de services }\end{array}$ & $\begin{array}{l}\text { Tiers secteur avec } \\
\text { importantes } \\
\text { inégalités } \\
\text { territoriales }\end{array}$ & $\begin{array}{l}\text { Essentiellement } \\
\text { tiers secteur et } \\
\text { marginalement } \\
\text { marché }\end{array}$ & $\begin{array}{l}\text { Services publics et } \\
\text { prestations en nature }\end{array}$ \\
\hline
\end{tabular}

Source : Martin, 2006.

- Dans le modèle libéral: le principe dominant de solidarité est le marché, pour préserver la responsabilité individuelle, auquel s'ajoute la solidarité familiale, complétés par l'assistance pour les plus pauvres, avec une forte stigmatisation. Les personnes âgées qui en ont la possibilité peuvent se protéger contre ce risque en recourant à la désépargne (la vente de son patrimoine immobilier principalement) et à l'assurance privée.

- Dans le modèle familialiste dominant dans les pays du sud de l'Europe, la famille reste le principe de solidarité privilégié, avec une obligation alimentaire qui pèse sur de larges pans du système de parenté. Les réponses complémentaires à cette aide de la parenté sont initiées au plan local, de manière très inégalitaire, principalement au niveau du tiers secteur et de l'action sociale bénévole.

- Dans le modèle corporatiste ou assurantiel, le principe dominant est la solidarité professionnelle, visant à donner au travailleur et à sa famille un niveau de vie et de rémunération indexé sur ses revenus antérieurs. La dépendance peut alors être conçue comme un nouveau "risque social" pouvant donner lieu à une nouvelle assurance sociale, financée par une cotisation et gérée dans le cadre du pacte social paritariste par les employeurs et les employés. La difficulté réside ici principalement dans la déconnexion entre le risque dépendance et les situations de travail. Le besoin de soin de longue durée se fait en général ressentir longtemps après la fin de l'activité professionnelle, ce qui pose des problèmes par rapport à la logique de ce système. Pour autant, ce modèle a été adopté avec quelques nuances en Allemagne et au Luxembourg.

- Dans le modèle social démocrate, le principe de solidarité repose sur la promotion pour chaque citoyen de droits sociaux à un ensemble de services publics permettant de faire face à ces situations de dépendance. Le financement est assuré par des impôts nationaux et locaux, gérés au plan des municipalités et donnant accès à des prestations de soins et d'aide en nature.

Si ces idéaux-types sont pertinents pour comprendre les variations des systèmes de protection sociale, en termes de projet de société, de conception du rôle de l'Etat, de pacte social, d'agencement ou de compromis politiques, etc., ils ne doivent pas masquer pour autant plusieurs phénomènes absolument cruciaux lorsque l'on aborde ces politiques sociales spécifiques : d'abord les disparités territoriales qui font que l'échelle nationale n'est pas forcément adaptée à une analyse de ce que recouvrent précisément ces dispositifs mis en œuvre à l'échelon territorial (pour un exemple de comparaisons territoriales voir Le Bihan \& Martin, 2006); ensuite, les variations importantes dans la compréhension de ces politiques en fonction des classes sociales concernées, du genre, ou des liens avec des questions d'immigration, de marché du travail, etc.; enfin, leur instabilité, c'est-à-dire le fait que les mesures adoptées évoluent rapidement. C'est sur ce dernier phénomène que nous proposons de nous pencher plus précisément.

La question est alors de savoir si ces évolutions infléchissent ou non les trajectoires des politiques proposées, remettant en cause les modèles traditionnels d'intervention de l'Etat. Jusqu'à quel point la conception universaliste du care est elle remise en cause dans les pays d'Europe du Nord? Dans 
quelle mesure s'éloigne-t-on d'un modèle familialiste dans les pays d'Europe du Sud ? Par rapport à ces différents idéaux types, le cas français apparaît comme un habile compromis, ne relevant pas exclusivement de l'un des modèles, mais les combinant au fur et à mesure des réformes et ajustements (Martin, Le Bihan, 2010c). La prochaine évolution du dispositif est d'ailleurs annoncée pour l'été 2011, l'objectif étant de développer la complémentarité avec le système assurantiel privé. Si les autres pays correspondent plus facilement aux différents types proposés, les récentes réformes montrent bien un glissement des logiques à l'œuvre: l'introduction d'une prestation monétaire nationale en Italie, qui permet l'externalisation d'une partie des tâches de care, ou à l'inverse la sollicitation de la famille, jusque-là considérée comme secondaire, dans les pays socio-démocrates.

\section{Vers une plus grande flexibilité des arrangements de care}

Les politiques engagées dans les pays européens s'organisent autour d'objectifs identiques qui peuvent apparaître paradoxaux - contenir les dépenses liées au développement d'une prise en charge publique de la dépendance tout en apportant une réponse aux familles qui ne peuvent pas faire face seules aux besoins croissants de leur(s) parent(s) âgé(s). L'analyse des systèmes, s'intéressant plus particulièrement aux évolutions des dispositifs depuis les années 1990, met en évidence un mouvement de convergence vers une recherche de flexibilité des arrangements de care, dans une logique de Welfare mix (Anttonen et al., 2003), combinant aide publique et aide familiale. La participation d'un aidant familial s'avère indispensable, même dans les pays traditionnellement attachés à une réponse avant tout publique aux besoins des populations, comme la Suède ou les Pays Bas. Favoriser la flexibilité pour aider les familles à mettre en place l'arrangement de care le plus adapté aux besoins de la personne âgée en tenant compte de la disponibilité ou non des membres de la famille, apparaît ainsi comme une solution permettant de concilier les différentes contraintes. L'analyse des mesures en faveur des personnes âgées dépendantes dans différents pays européens montre que cette recherche de flexibilité prend deux formes principales : I'introduction de prestations monétaires (Da Roit et Le Bihan, 2010), d'une part, de congés pour s'occuper de son parent âgé dépendant, d'autre part.

En Italie, comme en France, en Autriche, ou en Allemagne, mais aussi dans des pays où l'attribution d'une aide en services prévalait jusque là, comme les Pays Bas ou la Suède, les gouvernements ont fait le choix de créer des prestations monétaires versées aux personnes âgées dépendantes, en réponse à leur besoin d'aide quotidienne (tableau 4). L'un des principaux arguments avancé pour justifier ces mesures est le "libre choix " pour les familles, que faciliterait le cash. Quelles que soient les caractéristiques de la prestation, il revient aux familles de choisir les intervenants, professionnels ou non, publics ou privés, formels ou informels, qui viendront au domicile de la personne âgée. Le succès de la prestation sous forme de cash en Allemagne, où le système assurantiel laisse aux familles le choix entre une aide sous la forme de services ou de cash $^{3}$, témoigne du souhait des familles de pouvoir organiser elles-mêmes l'accompagnement du parent âgé, sans se voir imposer le recours à tel ou tel service, à telle ou telle intervenante.

${ }^{3}$ D'après les statistiques, jusqu'à présent plus de $70 \%$ des bénéficiaires de l'assurance dépendance en Allemagne préfèrent l'aide financière à l'aide en services, même si cette dernière donne accès à des services dont le coût est plus important que le montant attribué en cash. Ainsi, pour un niveau de dépendance 1 (niveau le plus faible), l'usager recevra $235 €$ s'il choisit le cash, alors que s'il choisit une aide en service, le montant correspondant est de $450 €$ (Burau et al., 2007). 
Mais les enjeux soulevés par les dispositifs de cash for care ne se limitent pas à cette liberté de choix octroyée en réponse à une demande des familles. La réalité est beaucoup plus complexe, le choix plus ambigu et les dispositifs en question très divers. Pour comprendre le sens insufflé à la politique à travers l'introduction de tels dispositifs, il est d'abord indispensable de les resituer dans le contexte social et historique de la politique menée. Ainsi, dans un pays comme les Pays Bas, l'introduction d'une prestation monétaire vise à diminuer les coûts de la prise en charge en flexibilisant le système existant et en responsabilisant les familles. A l'inverse, en France, en Allemagne ou en Italie, le glissement s'opère en sens inverse : dans ces pays traditionnellement familialistes, l'introduction de prestation monétaire témoigne au contraire du souci de soulager les familles. Le mode de régulation de ces dispositifs - c'est-à-dire leurs critères d'éligibilité et les conditions de leur utilisation - est un autre élément déterminant, car si la multiplication des prestations monétaires est un indice fort de convergence, leur régulation varie d'un pays à l'autre, introduisant des conceptions différentes de l'accompagnement des personnes âgées dépendantes. Ces variations ne sont pas anodines et ont des conséquences importantes sur le type de prise en charge adopté. L'analyse détaillée de la situation dans quatre pays (les Pays Bas, la France, l'Allemagne et l'Italie), représentant les différents régimes d'Etat Providence, permet de préciser ces variations (tableau 3).

Tableau 3 : Exemples de dispositifs de cash for care dans quatre pays européens

\begin{tabular}{|c|c|c|c|c|c|}
\hline & Allocation & Bénéficiaires & $\begin{array}{l}\text { Critères } \\
\text { d'attribution }\end{array}$ & Montant & Utilisation \\
\hline Pays Bas & $\begin{array}{l}\text { Persoonsgebonden } \\
\text { budget (PGB) }\end{array}$ & $\begin{array}{l}\text { Personnes } \\
\text { dépendantes }\end{array}$ & $\begin{array}{l}\text { En fonction du } \\
\text { niveau de } \\
\text { dépendance } \\
\text { En fonction du } \\
\text { niveau de revenu }\end{array}$ & $\begin{array}{l}\text { Budget moyen : } \\
11500 € / a n \text { en } \\
2006\end{array}$ & $\begin{array}{l}\text { Justification } \\
\text { des dépenses }\end{array}$ \\
\hline France & $\begin{array}{l}\text { Allocation } \\
\text { personnalisée } \\
\text { d'autonomie (APA) }\end{array}$ & $\begin{array}{l}\text { Personnes } \\
\text { dépendantes } \\
\text { de } 60 \text { ans et } \\
\text { plus }\end{array}$ & $\begin{array}{l}60 \text { ans et plus } \\
\text { En fonction du } \\
\text { niveau de } \\
\text { dépendance } \\
\text { En fonction du } \\
\text { niveau de revenu }\end{array}$ & $\begin{array}{l}4 \text { nivx (/mois): } \\
\text { Jusqu'à } \quad 524,84 € \\
\text { Gir4 } \\
\text { Jusqu'à } \quad 787,26 € \\
\text { Gir3 } \\
\text { Jusqu'à } 1049,68 € \\
\text { Gir2 } \\
\text { Jusqu'à } 1224,63 \\
\text { Gir1 } \\
\text { Montant moyen : } \\
\text { 494€/mois }\end{array}$ & $\begin{array}{l}\text { Justification } \\
\text { des dépenses }\end{array}$ \\
\hline Allemagne & $\begin{array}{l}\text { Assurance } \\
\text { dépendance }\end{array}$ & $\begin{array}{l}\text { Personnes } \\
\text { dépendantes } \\
\text { intégrées au } \\
\text { système } \\
\text { assurantiel }\end{array}$ & $\begin{array}{l}\text { Fonction du niveau } \\
\text { de dépendance }\end{array}$ & $\begin{array}{l}3 \text { nivx (/mois) } \\
215 € \text { niveau } 1 \\
420 € \text { niveau } 2 \\
675 € \text { niveau } 3\end{array}$ & $\begin{array}{l}\text { Utilisation } \\
\text { libre et non } \\
\text { contrôlée }\end{array}$ \\
\hline Italie & $\begin{array}{l}\text { Indennita di } \\
\text { Accompagnamento }\end{array}$ & $\begin{array}{l}\text { Personnes } \\
\text { dépendantes }\end{array}$ & $\begin{array}{l}\text { Personnes } \\
\text { dépendantes avec } \\
100 \% \text { d'incapacité } \\
\text { et/ou besoin d'une } \\
\text { surveillance } \\
\text { continue }\end{array}$ & $\begin{array}{l}\text { Montant } \\
\text { forfaitaire : } \\
472 € / \text { mois }\end{array}$ & $\begin{array}{l}\text { Utilisation } \\
\text { libre et non } \\
\text { contrôlée }\end{array}$ \\
\hline
\end{tabular}


Il est possible d'identifier trois principaux critères d'attribution des prestations financières : l'âge, le niveau de besoin et le niveau de revenu. Comme le décline le tableau 4, ces trois critères ne sont pas mobilisés dans tous les pays. Ainsi, seule la France utilise le critère d'âge -60 ans et plus - pour identifier les bénéficiaires de l'Allocation personnalisée d'autonomie. Dans les trois autres pays, l'état de " dépendance » est le seul élément déterminant. Ainsi en Italie, l'Indennita di Accompagnamento, initialement conçue pour les personnes handicapées et étendue aux personnes âgées dépendantes dans les années 1980, est destinée aux personnes souffrant d'un niveau d'incapacités important (100\%); elle concerne donc les personnes âgées les plus dépendantes. Dans les autres pays, différents niveaux de dépendance ou de besoin sont évalués, permettant de faire varier le montant financier alloué et de toucher les personnes les plus dépendantes, mais aussi celles qui sont de dépendance moyenne. Le dernier critère repéré, le niveau de revenus, ne concerne pas tous les pays d'Europe. Dans les exemples donnés (tableau 3), il n'est pas pris en compte en Italie et en Allemagne, où les montants sont forfaitaires, alors qu'en France et aux Pays Bas, les sommes attribuées sont diminuées en fonction du niveau de revenus, selon une logique de co-paiement.

Les possibilités d'utilisation des prestations et le contrôle de cet usage constituent un dernier élément de variation du mode de régulation (Da Roit, Le Bihan, 2010). Les usagers ont en effet la possibilité, lorsqu'une telle offre existe sur le marché, d'acheter des services, mais ils peuvent également, suivant certaines conditions, rémunérer un proche pour effectuer les tâches de soins ou se procurer le service sur le marché du travail au noir. Présenté explicitement comme un soutien financier aux familles en Italie, en Allemagne ou en Autriche, la prestation, dont le montant est insuffisant pour couvrir le besoin d'aide réelle, est alors conçue comme un revenu supplémentaire, intégré dans le revenu global du ménage. Elle est considérée comme une compensation symbolique pour l'aidant familial ou peut financer une partie des services nécessaires. L'utilisation de la prestation n'étant pas contrôlée, les familles peuvent recruter à moindre coût un aidant non professionnel sur le marché noir. Ce phénomène est particulièrement important en Italie, où l'on compte entre 650000 et 800000 badenti (Da Roit et Castegnaro, 2006), travailleurs venus des pays d'Europe centrale et de l'est, qui vivent avec les personnes âgées dépendantes et assurent pour 900 à $1200 € /$ mois une aide et une surveillance $24 \mathrm{~h} / 24$. Les dispositifs sont beaucoup plus régulés en France ou aux Pays Bas, car les prestations financières doivent être utilisées pour financer un besoin d'aide précis évalué par des professionnels du secteur médico-social. Libre ensuite à la personne âgée de choisir son intervenant parmi ses proches (à l'exception du conjoint en France) ou auprès de services professionnels, mais dans un cadre contrôlé puisque les bénéficiaires doivent justifier leurs dépenses, ce qui réduit les possibilités de développement d’un marché noir du care.

La régulation du dispositif de cash for care pose enfin la question de l'intervention ou non de professionnels dans l'organisation de l'arrangement de care. En d'autres termes, qui évalue le besoin d'aide des personnes âgées ? Qui assure le suivi de cette organisation susceptible d'être ajustée à mesure que la situation de dépendance évolue ? Qui prend en charge les tâches de coordination des différentes ressources, formelles et informelles, mobilisées au quotidien autour de la personne âgée ? Dans un pays comme l'Italie, où la régulation de la prestation est faible, ce rôle est laissé aux familles. Ce sont en effet les aidants familiaux qui apprécient le besoin d'aide de leur parent âgé, recrutent des intervenants extérieurs, professionnels ou non, et s'assurent au quotidien que tout se passe bien. Si les aidants assurent également une partie de ces fonctions dans les autres pays, ils ont en France ou aux Pays Bas notamment, des interlocuteurs professionnels dans le cadre de la 
procédure d'attribution et de suivi de la prestation, et ils peuvent également solliciter l'avis des responsables de services à domicile dont le rôle devient de plus en plus important (Campéon, Le Bihan, 2006).

Tenir compte des évolutions du marché du travail et du lien avec l'investissement dans le rôle d'aidant est posé par certains chercheurs comme un enjeu majeur des politiques en faveur des personnes âgées dépendantes (Haynes et al, 2010). La création de congés destinés aux aidants familiaux qui ont une activité professionnelle s'inscrit dans cette perspective et constitue une deuxième réponse des pouvoirs publics pour favoriser la flexibilité des arrangements de care. II s'agit cette fois de faciliter la conciliation vie familiale-vie professionnelle, en permettant aux aidants de se dégager du temps pour s'occuper de leurs parents âgés dépendants, sans pour autant mettre en péril leur activité professionnelle.

Dans la vie quotidienne, les filles et fils qui s'occupent de leur(s) proche(s) dépendant(s), mais qui ont par ailleurs d'autres responsabilités (familiales et professionnelles) doivent assumer toute une série de tâches, même si une partie des soins est déléguée à des professionnels du secteur médico-social. Ils s'occupent bien souvent de la gestion des comptes, des courses et de la logistique en général. Ils assument une veille continue, s'assurant que tout va bien au quotidien, que les relations avec les intervenants professionnels sont bonnes et ajustent l'organisation au fur et à mesure de l'évolution de l'état de santé de leur parent. Ce sont eux également qui prennent les contacts avec les différents services, effectuent les démarches administratives et accompagnent leur(s) parent(s) chez le médecin ou à l'hôpital. Enfin, ils doivent faire face aux imprévus et aux situations d'urgence, fréquentes dans les trajectoires de personnes âgées dépendantes. L'existence de congés peut permettre aux aidants familiaux d'assumer ces différentes tâches, mais tout dépend des caractéristiques des dispositifs mis en place, qui varient d'un pays à l'autre: ils peuvent être rémunérés ou non rémunérés, courts ou longs, spécifiques au secteur de la dépendance ou de portée plus large (tableau 4).

Table 4: Care leaves and working adjustments in the four countries

\begin{tabular}{|l|l|l|}
\hline France & Care leaves & Working adjustments \\
\hline Netherlands & $\begin{array}{l}\text { Familial solidarity leave to accompany a dying } \\
\text { relative. Three months, renewable once. Unpaid. } \\
\text { In } 2009, \text { creation of a short paid leave fixed at } \\
€ 47 / \text { day for three weeks to care for a dying relative } \\
\text { (3 weeks only). }\end{array}$ & $\begin{array}{l}\text { Working time reduction (RTT): 35h hours } \\
\text { of work /week }\end{array}$ \\
& $\begin{array}{l}\text { Emergency leave for unexpected personal family to work part time } \\
\text { problems } \\
\begin{array}{l}10 \text { days' leave/year to take care of a relative when } \\
\text { the person is the main carer. Paid 70\% of salary. } \\
\text { Long-term care leave: } 12 \text { unpaid weeks to take } \\
\text { care of a very sick close relative (child, parent) }\end{array}\end{array}$ & Adjustment of Hours Law \\
\hline
\end{tabular}




\begin{tabular}{|l|l|l|}
\hline Italy & $\begin{array}{l}\text { 3 days of paid leave/month for care of a severely } \\
\text { disabled person. The health commission has to } \\
\text { certify that the person is } 100 \% \text { disabled. }\end{array}$ & Possibility to work part time \\
\hline Portugal & $\begin{array}{l}15 \text { days of unpaid leave to take care of an elderly } \\
\text { parent }\end{array}$ & Possibility to work part time \\
\hline
\end{tabular}

Source: Martin, Le Bihan, à paraître.

Ces différentes caractéristiques ont un impact sur l'usage des congés. Au Portugal par exemple, où les congés sont possibles mais non rémunérés, les aidants familiaux préfèrent poser des jours de congés annuels lorsque cela est nécessaire. A l'inverse, les trois jours de congés rémunérés mensuels qui existent en Italie sont souvent utilisés par les familles (Da Roit, Le Bihan, 2009). La durée des congés proposés est également déterminante et il semble que la possibilité de prendre une journée de manière régulière soit une réponse adaptée à la demande des aidants familiaux. Les congés pour accompagner un proche en fin de vie, qui sont les plus répandus, sont certes utiles, mais ne permettent pas de faire face à l'ensemble des situations de dépendance. Enfin, des études qualitatives (Le Bihan, Martin, 2006 ; 2009) et quantitatives (Fontaine, 2009) ont montré que le congé long n'est pas forcément la réponse la plus adaptée à la demande des aidants familiaux, qui restent très attachés à leur activité professionnelle et la présente souvent comme nécessaire pour faire face aux responsabilités de care et éviter un investissement trop important. L'éventail des formules proposé aux Pays Bas apparaît dès lors comme la réponse la plus adaptée, mais elle n'existe pas dans autres pays, où les gouvernements ont opté pour l'une ou l'autre solution.

L'aménagement du temps de travail est une autre réponse pour faciliter la conciliation des temps. Mais si le temps partiel existe dans tous les pays étudiés, cela ne signifie pas que la pratique du temps partiel soit la même partout. Une fois encore, il faut distinguer le cas des Pays Bas, où les lois d'aménagement du temps de travail permettent aux familles de faire varier leur temps de travail en fonction des moments de la vie et des besoins (Knijn, 2001). Ainsi, les enfants de parents dépendants, comme les parents de jeunes enfants, n’hésitent pas à réduire leur temps de travail et/ou à l'organiser différemment, pour faire face à leurs différentes obligations.

\section{Conclusion}

Dans un contexte difficile de pression pour limiter les dépenses publiques, d'une part, de croissance de la demande de soutien des familles, d'autre part, la diversification des modalités d'intervention publique apparaît comme une réponse commune à l'ensemble des pays européens. Dans chacun des contextes nationaux étudiés, on constate en effet l'émergence d'une variété de dispositifs prestations monétaires, congés, aménagements du temps de travail - visant à ouvrir l'éventail des solutions de care possibles en facilitant la flexibilité dans l'organisation de la vie quotidienne du parent âgé dépendant. Le souci est bien d'articuler, dans une perspective de "Welfare mix" (Anttonen et al., 2003), les différentes sources de protection que sont la famille, les pouvoirs publics et le marché, ce qui permet de limiter l'investissement public et de satisfaire le désir des familles d'une plus grande liberté dans l'organisation des soins. Cependant, comme l'ont souligné nombre de théoriciens du care (Ungerson, 1997, 2005 ; Glendinning et Kemp, 2006), le risque est alors de donner trop de responsabilités aux familles, de limiter certes la dépense publique, mais d'amorcer un mouvement de familialisation / refamilialisation des activités de soins dispensés aux personnes âgées dépendantes. 
Comme le montre l'analyse de dispositifs comme les prestations monétaires, les congés ou l'aménagement du temps de travail, l'enjeu est alors celui de la régulation des dispositifs, qui sont plus ou moins encadrés et institutionnalisés, apportant un soutien plus ou moins important aux familles qui ne peuvent pas répondre seules aux besoins de leurs parents âgés dépendants. Ainsi, tout dépend dans la pratique des caractéristiques des dispositifs mis en place et du contexte politique plus général dans lequel ils s'inscrivent.

\section{Bibliographie}

Anttonen A., Baldock J., Sipila J.,(2003), The Young, the Old and the State. Social Care Systems in Five Industrial Nations, Cheltenham, UK, Northampton, MA, USA, Edward Elgar.

Bourdelais P., (1993), L'âge de la vieillesse. Paris, Odile Jacob.

Burau, V., Theobald, H., Blank, R.H, (2007), Governing Home Care. A cross-national comparison, Edward Elgar, Cheltenham, UK, Northampton, MA, USA.

Campéon A., Le Bihan B., (2006), "Les plans d'aide associés à l'Allocation personnalisée d'autonomie. Le point de vue des bénéficiaires et de leurs aidants ", Etudes et Résultats, $n^{\circ} 461$.

Da Roit, B. and Castegnaro, C. (2006), Chi cura gli anziani non autosufficienti, Milano: Angeli.

Da Roit, B. (2007), "Changing intergenerational solidarities within families in a Mediterranean welfare state", Current Sociology, 55: 251-69.

Da Roit B. et Le Bihan B., (2010), "Similar and Yet So Different: Cash for Care in six European Countries' Long Term Care Policies", Milbank Quarterly, vol. 88, n³, 2010, pp. 286-309.

Esping-Andersen G. (1999), Les trois mondes de l'Etat-providence. Paris, PUF (1 $1^{\text {ère }}$ édition chez Polity Press, 1990).

European Communities (2007), Europe's Demographic Future: Facts and Figures on Challenges and Opportunities. DG Employment, Social Affairs and Equal Opportunities.

Fontaine R., (2009), "Aider un parent âgé se fait-il au détriment de l'emploi? ", Retraite et Société, $n^{\circ} 58$, p. 32-59.

Glendinning G. and Kemp P.A. (eds), (2006) Cash and Care. Policy Challenges in the Welfare State. Bristol: Policy Press.

Grand C., Clément S., Bocquet H., (2000), " Personnes âgées », in Leclerc A., Fassin D., Grandjean H., Kaminski M., Lang T., Les inégalités sociales de santé. Paris, La Découverte.

Haynes P., Michael H. and Banks L., (2010) “Older People's Family Contacts and Long-Term Care Expenditure in OECD Countries: A Comparative Approach Using Qualitative Comparative Analysis", Social Policy and Administration, 44, 1: 67-84.

Knijn T., (2001) 'Care work: innovations in The Netherlands' in Daly, M. (ed.) Care work. The quest for security, Geneva: ILO, pp. 159-74. 
Le Bihan B., Martin, C. (2006), "A Comparative Case Study of care Systems for Frail Elderly People: Germany, Spain, France, Italy, United Kingdom and Sweden », Social Policy and Administration, vol. 40, n¹, p. 26-46.

Le Bihan, B. and Martin, C. (2007), "Cash for care in the French welfare state: a skilful compromise?" in C. Ungerson and S. Yeandle (eds), Cash for Care Systems in Developed Welfare States, London: Palgrave, pp.32-59.

Le Bihan, B., Martin, C. (2010a), "Vieillissement ", in D. Fassin et B. Hauray (dir), Santé publique. L'état des savoirs. Paris, La Découverte, p. 257-268.

Le Bihan B., Martin C. (2010b), "Quelles politiques pour la dépendance en Europe ? ", dans La dépendance des personnes âgées : quelle réforme ?, Regards sur l'actualité, $n^{\circ} 66$, La documentation française, décembre 2010, p. 61-73.

Le Bihan, B., Martin C., (2010c), «Reforming Long-term Care Policy in France: Towards a public / private complementarity ", Social Policy and Administration, vol. 44, n4, August 2010, pp. 392-410.

Martin C. (2006), "Prendre soin des personnes âgées dépendantes: Le défi européen et le modèle français", Revue Suisse de sociologie, vol. 32, 3, p. 7-21.

Robine J.M et Cheung S.L.K., (2008), " Nouvelles observations sur la longévité humaine ", Revue économique, vol.59, $\mathrm{n}^{\circ} 5$.

C. Ungerson (2005). Gender, labour markets and care work in five European funding regimes", in Pfau-Effinger B. and Geissler B., Care and Social Integration in European Societies (Bristol: Policy Press) p. 47-71. 